\title{
Tuberkulose und Rauchen
}

\author{
Tuberculosis and Tobacco Smoking
}

Autoren

Institute
R. Loddenkemper ${ }^{1}$, M. Brönnecke ${ }^{1}$, S. Castell ${ }^{1,2}$, R. Diel ${ }^{1,3}$

${ }^{1}$ Deutsches Zentralkomitee zur Bekämpfung der Tuberkulose, Berlin

${ }^{2}$ Abteilung für Epidemiologie, Helmholtz-Zentrum für Infektionsforschung, Braunschweig

${ }^{3}$ Lungenclinic Grosshansdorf, Großhansdorf, Deutsches Zentrum für Lungenforschung (DZL),

Universitäres Lungenzentrum Nord (ULZN) eingereicht $\quad 7.10 .2015$ akzeptiert nach Revision 16.11.2015

\section{Bibliografie}

Dol http://dx.doi.org/ 10.1055/s-0041-109601 Pneumologie 2016; 70: 17-22 (c) Georg Thieme Verlag KG Stuttgart · New York ISSN 0934-8387

\section{Korrespondenzadresse Prof. Dr. med. Robert Loddenkemper} Deutsches Zentralkomitee zur Bekämpfung der Tuberkulose Helios Klinikum Emil von Behring

Lungenklinik Heckeshorn Walterhöferstr. 11 14165 Berlin rloddenkemper@dzktuberkulose.de

\section{Zusammenfassung \\ $\nabla$}

Weltweit treten jährlich zirka 9,6 Millionen Neuerkrankungen und 1,5 Millionen Todesfälle durch Tuberkulose (TB) auf. Hierbei führt Rauchen als unabhängiger Risikofaktor etwa zu einer Verdoppelung des relativen Risikos nicht nur für die aktive Erkrankung, sondern auch für die latente TBInfektion und die Sterblichkeit an TB. Nach einer Hochrechnung werden bis zum Jahr 2050 durch das Rauchen vermutlich 18 Millionen Neuerkrankungen an TB zusätzlich verursacht, womit das TB-Eliminierungsziel der WHO infrage gestellt würde. Maßnahmen zur Raucherentwöhnung während und nach der Therapie, die in Tuberkuloseprogrammen bislang unzureichend beachtet werden, sind daher dringend erforderlich.

\section{Einleitung \\ $\nabla$}

Tuberkulose (TB) ist in vielen Regionen der Welt eines der größten gesundheitlichen Probleme mit geschätzten etwa 9,6 Millionen Neuerkrankungen und 1,5 Millionen Todesfällen pro Jahr [1]. Tabakrauchen ist die größte vermeidbare globale Krankheitsursache, die für den vorzeitigen Tod von jährlich fast 6 Millionen Menschen verantwortlich ist [2]. Eine Vielzahl von Veröffentlichungen hat in den letzten Jahren gezeigt, dass zwischen diesen beiden Epidemien ein enger Zusammenhang besteht; drei Metaanalysen aus dem Jahre 2007 (Slama et al. [3], Bates et al. [4] und Lin et al. [5]) kamen zu der Schlussfolgerung, dass Rauchen das Risiko für eine latente TB-Infektion, für eine aktive TB-Erkrankung und für den Tod an TB etwa verdoppelt. Rauchen als Risikofaktor ist in den TB-Kontrollprogrammen inzwischen zwar berücksichtigt [6,7], wird aber bislang nur unzureichend beachtet $[8,9]$.

Wie stark der Einfluss des Rauchens auf die TBEpidemiologie sein kann, wird in einer Hochrechnung aus dem Jahr 2011 gezeigt [10]. Danach

\section{Abstract \\ $\nabla$}

Worldwide there are annually about 9.6 million new cases and 1.5 million deaths due to tuberculosis (TB). Smoking is an independent risk factor causing approximately a twofold increase not only in active Tb disease but also in latent TB infection and mortality. In a mathematical model it is estimated that smoking would produce until 2050 an excess of 18 million tuberculosis cases from TB which would challenge the TB elimination goal of the WHO. Smoking cessation methods during and after TB treatment, which at present are insufficiently included into TB programmes, are urgently needed.

wird das Rauchen zwischen 2010 und 2050 unter den derzeitigen Rauchertrends global im Vergleich zu den Vorhersagen der WHO, die den Einflussfaktor Rauchen nicht berücksichtigen, zu zusätzlichen 18 Millionen TB-Neuerkrankungen (Anstieg der TB um 7\% von 256 Millionen auf 274 Millionen) führen. Bei der hieraus resultierenden erhöhten Zahl von Todesfällen ist der mögliche Einfluss von HIV und der tabakassoziierten COPD noch nicht eingerechnet [11].

In dieser Übersicht soll anhand der aktuellen Literatur der Zusammenhang zwischen TB und Tabakkonsum unter verschiedenen Aspekten Infektion mit dem TB-Erreger, Erkrankung an TB, Einfluss auf Therapieerfolg einschließlich Rezidivgefahr und Mortalität sowie Tabakentwöhnung dargestellt werden. 


\section{Historisches}

$\nabla$

In Deutschland gab es schon zu Beginn des vorigen Jahrhunderts Hinweise auf den ursächlichen Zusammenhang zwischen TB und Tabak. So veröffentlichte Ludolph Brauer im ersten Heft der von ihm seit 1903 herausgegebenen Beiträge zur Klinik der Tuberkulose einen umfangreichen Artikel, in welchem er das gehäufte Auftreten der TB bei Arbeitern in Zigarrenfabriken im Vergleich zur übrigen Bevölkerung untersuchte [12]. Er beschrieb, dass mit steigender Produktion auch mehr Menschen an der TB sterben, was auf eine „Beziehung beider Faktoren zueinander“ hindeute.

In der Zeitschrift „Der Tabakgegner“ findet sich 1913 die Forderung für ein neues Epidemie-Gesetz in Österreich, wonach darin auch die Gefahren des Rauchens berücksichtigt werden sollten mit der Begründung: „Es ist keine Übertreibung, wenn man behauptet, dass seit Duldung der Tabakunsitte die Erkrankungen an Lungentuberkulose sich mehr als verzehnfacht haben“ [13].

Fritz Lickint wies in seiner 1939 veröffentlichten Monografie „Tabak und Gesundheit“ ausführlich auf die negativen Auswirkungen des Tabakkonsums auf die TB-Infektion und den Verlauf der TB-Erkrankung hin, u.a. mit dem Zitat einer im Volksmund verbreiteten Redensart, „daß sich jemand die Schwindsucht angeraucht habe“ [14]. In seinem 1940 erschienenen Buch „Tabakgenuss und Gesundheit“ postuliert Lickint ein erhöhtes Risiko für Raucher, eine (Kehlkopf-) Tuberkulose zu entwickeln, bedingt durch die in der Atemwegschleimhaut ablaufende chronische Entzündungsreaktion. Er berichtet über schlechtere Behandlungserfolge bei Rauchern mit Tuberkulose und einer sich günstiger entwickelnden Krankheitsprognose unter Tabakkarenz und schlussfolgert, dass ein Rauchstopp allen TB-Kranken ärztlich anzuraten sei [15].

Vorstand und Beirat der Deutschen Tuberkulose-Gesellschaft, der heutigen Deutschen Gesellschaft für Pneumologie und Beatmungsmedizin (DGP), forderten bereits 1939, dass in allen Lungenheilstätten und Tuberkulosekrankenhäusern ein absolutes Rauchverbot in allen dienstlichen Räumen sowohl für die Patienten als auch für die Angestellten einzuhalten sei [16].

In den USA machte G. B. Webb im Jahre 1918 auf die negativen Effekte des Zigarettenrauchs im Zusammenhang mit der TB aufmerksam [17]. Dort wurde schon zu Beginn des vorigen Jahrhunderts das Spucken als Folge des Tabakkauens wegen der damit verbundenen Gefahr der TB-Ansteckung untersagt. Tabakkauen war davor beliebt und gerade diese Hygienemaßnahmen zur Prävention der TB könnten ironischerweise der Ausbreitung des Rauchens Vorschub geleistet haben: Mit der damals aufkommenden Furcht, dass der ausgespuckte Kautabak wesentlich zur TBVerbreitung beitragen könnte, wurde das Tabakkauen zunehmend verpönter und brachte das inhalative Rauchen zunächst in den USA, später in Europa in Mode [18-20]. Dieses gesetzliche „Spuck“-Verbot in den USA trug demnach wesentlich dazu bei, dass innerhalb eines Jahrzehnts das Zigarettenrauchen das Tabakkauen als wesentliche Quelle von Nikotin überholt hatte [21,22].

Zunächst wurde aber die allgemeine Einstellung zum Rauchen als Einflussfaktor auf die Tuberkulose vor allem durch zwei Arbeiten aus den 1960er-Jahren beeinflusst [23,24]. Diese hatten den Zusammenhang zwischen Tuberkulose, Tabak- und Alkoholkonsum untersucht und Alkohol als den Hauptrisikofaktor benannt. In der Folge galt als „Expertenmeinung“, dass Rauchen nicht mit TB assoziiert sei. Rauchen als Gesundheitsproblem betraf zudem über lange Zeit hauptsächlich nur die Industrienationen, in de- nen gleichzeitig die Tuberkulosezahlen zurückgingen. Die sogenannten Entwicklungsländer mit hohen und/oder ansteigenden Tuberkuloseinzidenzen blieben zunächst bis in die neuere Zeit vom Rauchen weitgehend verschont [25]. Der Zusammenhang zwischen Tabakrauchen und Tuberkulose wurde so bis fast in die 1990er-Jahre von Public Health-Institutionen [26] wie vonseiten der Kliniker [27] zumeist übersehen oder nur als zufällig erachtet $[28,29]$. Erst im letzten Jahrzehnt wurde der Assoziation von Rauchen und TB weitreichendere Aufmerksamkeit zuteil [30].

\section{Ätiologischer Zusammenhang zwischen Rauchen und TB $\nabla$}

Als Erklärung dafür, dass das Rauchen ein wichtiger Risikofaktor für die TB ist, werden verschiedene Faktoren, vor allem eine Beeinträchtigung der lokalen und allgemeinen Immunabwehr und eine Störung der bazillären Klärfunktion im Bronchialsystem, diskutiert [31 - 33]. Bekannt ist, dass beim Raucher die mukoziliäre Clearance eingeschränkt ist [34], wodurch den TB-Bakterien das Vordringen in die Alveolen erleichtert werden kann [5]. Der Tabakrauch beeinträchtigt zudem die Funktion der Alveolarmakrophagen (AM), die ein wichtiger Faktor im Abwehrmechanismus gegen Bakterien sind [36]. Aus den Lungen von Rauchern isolierte AM haben im Vergleich zu denen von Nichtrauchern eine herabgesetzte Fähigkeit zur Phagozytose und liefern eine geringere Menge an sezernierten proinflammatorischen Zytokinen [37]. AM von Rauchern konnten das intrazelluläre Wachstum von M. tuberculosis (Mtb) nicht kontrollieren. Die AM von Nichtrauchern dagegen produzieren nach Infektion mit Mtb signifikant mehr Tumornekrosefaktor-Alpha, IFN-gamma und IL-1beta im Vergleich mit nicht-infizierten Makrophagen. Die Lunge von Rauchern enthält zudem mehr AM, welche die immunsuppressiv wirkenden FoxP3 T-Zellen induzieren [38, 39]. Nachgewiesen wurde auch, dass Zigarettenrauch die T-Zell-Antwort auf das TBBakterium verringert [40]. Ein weiterer möglicher Mechanismus könnte in der direkten Wirkung von Nikotin auf die Nikotinrezeptoren liegen, wodurch die intrazelluläre Produktion von Tumornekrosefaktor-Alpha im AM herabgesetzt und damit die Eindämmung von Mtb eingeschränkt wird [41].

\section{Risiko für eine TB-Infektion \\ $\nabla$}

Bei Untersuchungen zum Zusammenhang zwischen Rauchen/ Passivrauchen und Tuberkulose ist immer die Möglichkeit weiterer gleichzeitig bestehender Risikofaktoren wie Alkohol- und Drogenkonsum, Mangelernährung, Immunschwäche, oder Sozialstatus als „Confounder“ zu beachten. Bei Studien zum Zusammenhang zwischen Rauchen/Passivrauchen und TB-Infektion kommt noch hinzu, dass es keinen eindeutig sicheren Nachweis einer latenten TB-Infektion gibt, auch wenn Tuberkulinhauttest (THT) und Interferon Gamma-Release-Assays (IGRAs) eine relativ hohe Sensitivität und Spezifität besitzen [8].

Einzelne Studien aus den Jahren 1997-2005 hatten bereits gezeigt, dass das Ansteckungsrisiko mit der Menge und Dauer des Rauchens steigt [42-45]. Die drei unabhängig voneinander durchgeführten Metaanalysen aus dem Jahr 2007 hatten ergeben, dass die Exposition gegenüber Tabakrauch das Risiko einer TB-Infektion etwa verdoppelt. Die Metaanalyse von Slama et al. [3], bei der 5 von 53 eingeschlossenen Arbeiten die Beziehung 
zwischen Rauchen und Infektion untersuchten, belegt einen signifikanten Einfluss von aktivem Tabakrauchen auf die Entstehung einer TB-Infektion (Odds Ratio [OR]) 1,8, 95\% CI [Konfidenzintervall] 1,5-2,1). Bates et al. [4] fanden, dass die Wahrscheinlichkeit für eine Infektion nach Kontakt mit einem Patienten mit offener Lungentuberkulose bei Tabakrauchern auf das 1,7-Fache erhöht ist. Ähnliche Wahrscheinlichkeiten fanden sich in der dritten Metaanalyse (Lin et al. [5]). In der Metaanalyse der WHO und der IUATLD konnten alle acht Studien, die das Infektionsrisiko unter Tabakeinfluss untersuchten, den schädlichen Effekt des Tabakrauchens belegen; einige fanden eine Dosis-Wirkungs-Beziehung: höhere Odds Ratio für steigenden Zigarettenkonsum und mehr Raucherjahre [6].

Eine kürzlich veröffentlichte Studie aus den USA [46], welche die Tabakrauchexposition durch Cotinin-Bestimmung im Speichel kontrollierte, fand, dass erwachsene Raucher gegenüber Nichtrauchern ein erhöhtes Risiko für eine LTBI haben (AOR [adjustierte OR] 2,31; 95\% CI 1,17-4,55). Erwachsene Passivraucher hatten zwar erhöhte Odds, jedoch war dies statistisch nicht signifikant. Dagegen zeigte sich bei im Ausland geborenen Erwachsenen sowohl für aktives (AOR 2,56; 95\% CI 1,20-5,45) als auch passives Rauchen (AOR 2,27; 95\% CI 1,09-4,72) ein signifikant erhöhtes Risiko. Bei Kindern war in dieser Studie kein Zusammenhang zu Aktiv- oder Passivrauchen zu eruieren. Während eine aktuelle Metaanalyse nur eine weiche Assoziation zwischen Passivrauchen und TB-Infektion aufzeigte [47], konnte in einer spanischen Studie gezeigt werden, dass das Risiko einer Übertragung des TB-Erregers bei Kontakten mit rauchenden TB-Patienten höher als bei nicht rauchenden TB-Patienten ist; aufgrund der Ergebnisse wurde geschätzt, dass Passivrauchen für 12,8\% der TB-Infektionen verantwortlich war [48]. Bei Kindern fand sich in einer peruanischen Studie, dass der Kontakt mit rauchenden TB-Patienten im selben Haushalt das Risiko einer Übertragung des TB-Erregers gegenüber Haushalten mit nicht rauchenden TB-Patienten verdoppelt [49]. Auch werden Maßnahmen gefordert, die dem Schutz von nichtrauchenden TB-Patienten vor Passivrauch dienen sollen [50].

\section{Risiko für eine aktive TB-Erkrankung}

$\nabla$

Die drei bereits erwähnten Metaanalysen aus dem Jahr 2007 kamen auch zu dem Ergebnis, dass aktive Raucher ein mehr als doppelt so hohes Risiko haben, an einer TB zu erkranken $[3,4,5]$ : Slama et al. [3] fanden in 21 von 24 Studien einen Zusammenhang zwischen Rauchen und einem erhöhten Erkrankungsrisiko für eine Lungentuberkulose. Aktive Raucher haben danach eine mehr als doppelt so hohe Chance zu erkranken (OR 2,6; 95\% CI $2,1-3,4)$. Sogar ehemalige Raucher sind verglichen mit Nichtrauchern noch um das 1,6-Fache (OR; $95 \% \mathrm{CI}$ : 1,2 - 2,1) gefährdet [3]. Die Forschergruppe um Bates wies ein 2,3 bis 2,7-fach erhöhtes relatives Risiko bei 13 eingeschlossenen Studien nach [4]. Lin et al. [5] bestätigten diese Ergebnisse, wobei die Evidenzlage für die pulmonale TB größer als für die extrapulmonale TB war. Eine spanische Querschnittsstudie an mehr als 13000 TB-Patienten erbrachte, dass bei Rauchern im Vergleich mit Nichtrauchern eine um $50 \%$ höhere Wahrscheinlichkeit besteht, eine pulmonale TB und zudem fast zweimal so häufig eine ausgedehntere Form mit Kavernen zu entwickeln [51].

Abgesehen vom Rauchen hat auch der sozioökonomische Status mit Faktoren wie Ernährung, Unterkunft und anderen Gefährdungen Einfluss auf das Risiko einer TB-Erkrankung. Bei der In- terpretation der obigen Ergebnisse muss deshalb die unvollständige Berücksichtigung potenzieller Einflussgrößen beachtet werden. Allerdings wurden die prospektiven Kohortenstudien von Jee et al. [52] und Lin et al. [53] im Hinblick auf Alter, Geschlecht und Alkoholkonsum kontrolliert durchgeführt. Dass auch Passivrauchen eine erhöhte Gefahr für die Entwicklung einer TB bedeutet, zeigte sich in einer prospektiven Studie aus Hongkong [54], die an einer großen Kohorte von mehr als 15000 weiblichen Nierauchern im Alter zwischen 65 und 74 Jahren durchgeführt worden war: Hier war die Exposition im Haushalt mit der Entwicklung einer aktiven TB (Hazard Ratio [HR] 1,49; 95\% CI 1,01 - 2,19) und einer kulturell bestätigten TB (HR 1,70; 95\% CI 1,04-2,80) verbunden. Obwohl somit die Forschung eine starke Assoziation zwischen Rauchen (inklusive Passivrauchen) und Tuberkulose aufzeigt, ist noch ungeklärt, ob dies Ausdruck eines erhöhten Risikos für eine TB-Infektion oder für eine Reaktivierung zu einer aktiven TB-Erkrankung ist [13]. Vermutlich kommen beide Möglichkeiten in Frage.

\section{Risiko für Rezidiv einer TB \\ $\nabla$}

Dass Rauchen den Therapieerfolg negativ beeinflusst, konnte ebenfalls in einigen Studien gezeigt werden. So war die mikroskopische Sputumkonversion nach 2 Monaten Therapie in einer Studie in Kuwait [55] bei Rauchern mit weit fortgeschrittenen radiologischen Veränderungen oder deutlichem mikroskopischen Nachweis gegenüber Nichtrauchern statistisch signifikant verzögert. In einer brasilianischen Studie [56] wurde bei Rauchern mit einer nicht-kavernösen Tuberkulose eine Verzögerung der kulturellen Sputumkonversion nach 2-monatiger Therapie beobachtet. Ähnliche Ergebnisse erbrachte eine weitere Studie in Brasilien [57], worauf die Empfehlungen beruhen, die Patienten noch während der Therapie vom Rauchen zu entwöhnen. Auch erfolgreich behandelten Patienten ist eine Fortführung des Rauchverzichts nach Beendigung der Tuberkulosetherapie anzuraten. Dafür sprechen die Daten einer weiteren brasilianischen Studie [58], die mehr als 1000 Tuberkulosepatienten einschloss. Von den 754 als erfolgreich behandelt geltenden TB-Erkrankten wurden 711 über einen Zeitraum von drei Jahren nachbeobachtet; bei 37 (5,2\%) kam es zu einem Rezidiv. Die Studie konnte belegen, dass bei aktivem Rauchen die Gefahr eines TB-Rezidivs um das 2,5-Fache ansteigt (OR 2,53; $95 \%$ CI 1,23-5,21).

Auch andere Studien haben das höhere Wiedererkrankungsrisiko für Raucher bestätigt $[59,60]$. In der koreanischen Studie von Jee et al. erhöhte Rauchen das Risiko eines Rezidivs bei Männern um 30\%, jedoch nicht bei Frauen [52]. In einer Studie aus Taiwan von Yen et al. [61] an über 5500 (zunächst) erfolgreich behandelten TB-Patienten war das Rauchen von täglich mehr als 10 Zigaretten mit einer doppelt so hohen Rezidivrate als der bei Nichtrauchern verbunden. Die bislang größte prospektive Studie an über 16000 TB-Patienten in Hongkong von Leung et al. [62] erbrachte bei Rauchern nicht nur einen schlechteren Therapieerfolg, sondern auch ein erhöhtes Rezidivrisiko mit einem deutlichen Gradienten vom Nierauchern zu Ex-Rauchern (7,2\%) und aktiven Rauchern (12,2\%). Die Autoren weisen darauf hin, dass bei Wiederauftreten einer TB-Erkrankung hier nicht sicher zwischen einem Rezidiv und einer Reinfektion, die bei Rauchern ebenfalls eine größere Gefahr darstellt, unterschieden werden konnte. 
Tab. 1 Zusammenhänge zwischen Rauchen und dem relativen Risiko einer latenten TB-Infektion, der Entwicklung einer Erkrankung und der Sterblichkeit an aktiver TB (adaptiert von Zyl Smith et al., Eur Respir ] 2010 [32]).

\section{Gepooltes Relatives Risiko (95\% Cl)}

\begin{tabular}{|llll|}
\hline Ergebnis & Salma et al. 2007 & Lin et al. 2007 & Bates et al. 2007 \\
\hline TB-Infektion & $\sim 1,8(1,5-2,1)$ & $\sim 1,7(1,5-2,8)$ & $\sim 1,7(1,5-2,0)$ \\
\hline TB-Erkrankung & $\sim 2,3(1,8-3,0)$ & $\sim 2,0(1,6-2,6)$ & $\sim 2,3(2,0-2,8)$ \\
\hline TB-Sterblichkeit & $\sim 2,28(1,3-3,7)$ & $\sim 2,0(1,1-3,5)$ & $\sim 2,1(1,4-3,4)$ \\
\hline
\end{tabular}

Abkürzung: $\mathrm{Cl}=$ Konfidenzintervall

\section{Risiko für Tod an TB}

$\nabla$

Bei aktiven Raucher besteht im Vergleich zu Nichtrauchern eine bis zu doppelt so hohe Gefahr, an TB zu versterben [59,63]. Von den drei o.g. Metaanalysen aus dem Jahr 2007 fand nur die Studie von Bates et al. kein höheres TB-Sterblichkeitsrisiko für Raucher [4].

Wie dargestellt gibt es Hinweise darauf, dass Rauchen die Schwere des Krankheitsverlaufs, die Geschwindigkeit der bakteriologischen Konversion, die Therapieversager- und Rückfallraten ungünstig beeinflussen kann. Damit lässt sich vermutlich auch das erhöhte Risiko, an Tuberkulose zu versterben, erklären. Weitere Studien sind jedoch notwendig, um belastbare Ergebnisse zu dieser Fragestellung zu erhalten. Weltweit könnte jeder fünfte Tod an Tuberkulose vermieden werden, wenn die Patienten keine Raucher wären [64].

\section{Tabakentwöhnung während und nach TB-Therapie \\ $\nabla$}

Die oben erwähnte Literatur, welche den erheblichen negativen Einfluss des Rauchens auf TB-Infektion, TB-Erkrankung und die Prognose aufzeigt hat, hat auch zu den Empfehlungen der WHO geführt, TB-Patienten zumindest während und nach der Behandlung vom Rauchen zu entwöhnen [6]. Tatsächlich gibt es einige Studien aus Entwicklungsländern mit hoher TB-Prävalenz, die die Auswirkungen der Tabakentwöhnung auf den Verlauf der TB untersucht haben [65]: Ein initiales Motivationsgespräch von 5 10 Minuten und kurze Erinnerungen bei jeder Visite führten in Indonesien zu einem Anstieg der Nichtraucher nach 6-monatiger Therapie von $86,1 \%$ im Vergleich zu initial 18,5\% Nichtrauchern in 2011 [66]. In Nepal beendeten 39\% einer Kohorte von 77 Rauchern das Rauchen binnen 6 Monaten nach einer kurzen Beratung jeweils zu Behandlungsbeginn, nach 2 und 5 Monaten [67]. In Südafrika führte ein kurzes Motivationsgespräch durch eine Krankenschwester nach 6 Monaten immerhin noch zu einer mindestens doppelt so hohen Rauchabstinenz (21,5\% der 205 neu erkrankten Erwachsenen versus 9,3\% der 204 Kontrollpatienten) [68].

Erstaunlich ist aber, dass es kaum Studien gibt, die die Auswirkungen der Tabakentwöhnung auf den Verlauf der TB untersucht haben. Zellweger et al. [69] zitieren lediglich eine Studie aus dem Sudan, die fand, dass TB-Patienten, die von in der Raucherberatung geübtem medizinischen Personal betreut wurden, davon deutlich profitierten: Bei Patienten, die bei der Entwöhnung mitmachten, wurde die Therapie in $83 \%$ regulär beendet im Vergleich zu 59\% bei den Patienten, die nicht in das Entwöhnungsprogramm eingeschlossen waren [70].

\section{Schlussfolgerungen}

Wie die in Tab. 1 dargestellten Ergebnisse der 3 Metaanalysen aus dem Jahr 2007 zeigen, kann es als gesichert gelten, dass sich durch Rauchen das Risiko für eine TB-Infektion, für eine aktive TB-Erkrankung und die Sterblichkeit an Tuberkulose etwa verdoppelt.

Daraus leiten sich zwingend die Empfehlungen zur Tabakentwöhnung sowohl während als auch nach der Behandlung einer TB ab $[65,69]$. Aber auch davon unabhängig muss das Rauchen generell eingedämmt werden, da es unter Berücksichtigung der aktuellen Trends nach einer Hochrechnung zwischen 2010 und 2050 global im Vergleich zu den Vorhersagen der WHO, die den Einflussfaktor Rauchen nicht berücksichtigen, zusätzlich zu 18 Millionen TB-Neuerkrankungen kommen könnte [10]. Eine stärkere politische Fokussierung dieser Problematik ist angesichts der End-TB-Strategie der WHO dringend erforderlich [71,72].

\section{Interessenkonflikt}

Die Autoren geben an, dass kein Interessenkonflikt besteht.

\section{Literatur}

1 World Health Organisation. Global tuberculosis report 2015. WHO/ HTM/TB/2015.22. Geneva: WHO; 2015

2 Mackay J, Schluger N. Global tobacco epidemic. In: Loddenkemper R, Kreuter M eds. The Tobacco Epidemic. ed 2, rev. and ext. Prog Respir Res. Basel: Karger; 2015; 42: 19-26

3 Slama $K$, Chiang $C Y$. Providing and monitoring quality service for smoking cessation in tuberculosis care. Int J Tuberc Lung Dis 2007; 11: $838-847$

4 Bates MN, Khaladina A, Pai M et al. Risk of tuberculosis from exposure to tobacco smoke. Arch Intern Med 2007; 167: 335-342

5 Lin HH, Ezzati M, Murray M. Tobacco smoke, indoor air pollution and tuberculosis: a systematic review and meta-analysis. PLoS Med 2007; 4: e20

6 World Health Organisation. A WHO/The Union monograph on TB and tobacco control: joining efforts to control two related epidemics. Geneva: WHO; 2007

7 Enarson DA, Slama K, Chiang CY. Providing and monitoring quality service for smoking cessation in tuberculosis care. Int J Tuberc Lung Dis 2007; $11: 838-847$

8 Diel R, Loddenkemper R, Zellweger JP et al. European Forum for TB Innovation. Old ideas to innovate TB control: preventive treatment to achieve elimination. Eur Respir J 2013; 42: 785-801

9 D'Ambrosio L, Dara M, Tadolini M et al. European national programme representatives. Tuberculosis elimination: theory and practice in Europe. Eur Respir J 2014; 43: 1410-1420

10 Basu S, Stuckler D, Bitton A et al. Projected effects of tobacco smoking on worldwide tuberculosis control: mathematical modelling analysis. BMJ 2011; 4: 343

11 van Zyl Smit RN, Pai M, Yew WW et al. Global lung health: the colliding epidemics of tuberculosis, tobacco smoking, HIV and COPD. Eur Respir J 2010; 35: 27-33

12 Brauer L. Das Auftreten der Tuberkulose in Cigarrenfabriken. Beiträge zur Klinik der Tuberkulose und spezifischen Tuberkulose-Forschung 1903; $1: 1-47$

13 Gesetzentwurf betreffend die Verhütung und Bekämpfung der Tuberkulose. Der Tabakgegner 1913; 2: 3

14 Lickint F. Tabak und Organismus. Handbuch der gesamten Tabakkunde. Stuttgart: Hippokrates-Verlag Marquardt \& Cie; 1939

15 Lickint F. Tabakgenuß und Gesundheit. Hannover: Bruno Wilkens Verlag; 1940: 49-53

16 Reichs-Tuberkulose-Ausschuß. Tuberkulose-Lexikon für Ärzte und Behörden. Leipzig: Thieme; 1943: 142 
17 Webb GB. The effect of the inhalation of cigarette smoke on the lungs. A clinical study. Am Rev Tuberc 1918; 1: 25-27

18 Snider GL, Doctor L, Demas TA et al. Obstructive airway disease in patients with treated pulmonary tuberculosis. Am Rev Respir Dis 1971; 103: $625-640$

19 Christen AG, Swanson BZ, Glover ED et al. Smokeless tobacco: the folklore and social history of snuffing, sneezing, dipping, and chewing. J Am Dent Assoc 1982; 105: 821 - 829

20 Bolinder G, Gilljam H. Smokeless tobacco - health hazards or less harm? In: Loddenkemper R, Kreuter M eds. The Tobacco Epidemic. ed 2, rev. and ext. Prog Respir Res. Basel: Karger; 2015; 42: 243-251

21 Kluger R. Ashes to ashes: America's hundred-year cigarette war, the public health, and the unabashed triumph of Philip Morris. New York: Vintage Books; 1997; zitiert in Leone FT, Douglas IS. The emergence of e-cigarettes: a triumph of wishful thinking over science. Ann Am Thorac Soc 2014; 11: 216-219

22 Behr J, Nowak D. Tobacco smoke and respiratory disease. Eur Resp Mon 2002; 21: $161-179$

23 Lewis JG, Chamberlain DA. Alcohol consumption and smoking habits in male patients with pulmonary tuberculosis. Brit J Prev Soc Med 1963; 17: $149-152$

24 Brown KE, Campbell AH. Tobacco, alcohol and tuberculosis. Brit J Dis Chest 1961; 55: 150-158

25 Zellweger JP. Tobacco and tuberculosis. Monaldi Arch Chest Dis 2008; 69: $83-85$

26 Yach $D$. Partnering for better lung health; improving tobacco and tuberculosis control. Int J Tuberc Lung Dis 2000; 4: 693-697

27 Maurya V, Vijayan K, Shah A. Smoking and tuberculosis; an association overlooked. Int J Tuberc Lung Dis 2002; 6: 942-951

28 Bates I, Fenton C, Gruber J et al. Vulnerability to malaria, tuberculosis, and HIV/AIDS infection and disease. Part 1: determinants operating at individual and household level. Lancet Infect Dis 2004; 4: 267-277

29 Bothamley GH. Smoking and tuberculosis: a chance or causal association? Possible explanations for the association between smoking and tuberculosis (Editorial). Thorax 2005; 60: 527-528

30 Slama K, Chiang C-Y, Enarson D. An educational series about tobacco cessation interventions for tuberculosis patients: what about other patients? Int J Tuberc Lung Dis 2007; 11: 244

31 Davies PD, Yew WW, Ganguly D et al. Smoking and tuberculosis: the epidemiological association and immunopathogenesis. Trans R Soc Trop Med Hyg 2006; 100: 291 - 298

32 Van Zyl Smit RN, Pai M, Yew WW et al. Global lung health: the colliding epidemics of tuberculosis, tobacco smoking, HIV and COPD. Eur Respir J 2010; 35: 27-33

33 U.S. Department of Health and Human Services, Centers for Disease Control and Prevention, National Center for Chronic Disease Prevention and Health Promotion, Office on Smoking and Health. The health consequences of smoking - 50 years of progress: a report of the Surgeon General. Atlanta GA: 2014

34 Houtmeyers E, Gosselink R, Gayan-Ramirez G et al. Regulation of mucociliary clearance in health and disease. Eur Respir J 1999; 13: 1177 1188

35 Ernst JD. The immunological life cycle of tuberculosis. Nat Rev Immunol 2012: $12: 581-591$

36 Shang $S$, Ordway $D$, Henao-Tamayo $M$ et al. Cigarette smoke increases susceptibility to tuberculosis - evidence from in vivo and in vitro models. J Infectious Dis 2011; 203: 1240-1248

37 Sopori $M$. Effects of cigarette smoke on the immune system. Nat Rev Immunol 2002; 2: $372-377$

38 O'Leary SM, Coleman MM, Chew WM et al. Cigarette smoking impairs human pulmonary immunity to Mycobacterium tuberculosis. Am J Respir Crit Care Med 2014; 190: 1430-1436

39 Van Zyl-Smit RN, Meldau R, Semple PL et al. Cigarette smoke impairs cytokine responses and BCG containment in alveolar macrophages. Thorax 2014; 69: $363-370$

40 Feng $Y$, Kong $Y$, Barnes PF et al. Exposure to cigarette smoke inhibits the pulmonary T-cell response to influenza virus and Mycobacterium tuberculosis. Infect Immun 2011; 79: 229-237

41 Wang $H$, Yu M, Ochani $M$ et al. Nicotinic acetylcholine receptor alpha7 subunit is an essential regulator of inflammation. Nature 2003; 421: $384-388$

42 Anderson RH, Sy FS, Thompson $S$ et al. Cigarette smoking and tuberculin skin test conversion among incarcerated adults. Am Prev Med 1997; 13: $175-181$
43 Den Boon S, Van Lill SWP, Borgdorff MW et al. The association between smoking and tuberculosis infection: a population survey in a high tuberculosis incidence area. Thorax 2005; 60: 555-557

44 Hussain H, Akthar S, Nanan D. Prevalence of and risk factors associated with Mycobacterium tuberculosis infection in prisoners, North West Frontier Province, Pakistan. Int J Epidemiol 2003; 32: 794-799

45 Plant AJ, Watkins RE, Gushulak B et al. Predictors of tuberculin skin test reactivity among prospective Vietnamese migrants: the effects of smoking. Epidemiol Infect 2002; 128: 37-45

46 Lindsay RP, Shin SS, Garfein RS et al. The association between active and passive smoking and latent tuberculosis infection in adults and children in the United States: Results from NHANES. PLoS ONE 2014; 9: e93137

47 Dogar OF, Pillai N, Safdar $N$ et al. Second-hand smoke and the risk of tuberculosis: a systematic review and a meta-analysis. Epidemiol Infect 2015; 29: $1-15$

48 Godoy P, Calya JA, Carmona G et al. Smoking in tuberculosis patients increases the risk of infection in their contacts. Int J Tuberc Lung Dis 2013; 17: 771 - 777

49 Huang CC, Tchetgen ET, Becerra MC et al. Cigarette smoking among tuberculosis patients increases risk of transmission to child contacts. Int J Tuberc Lung Dis 2014; 18: 1285 - 1291

50 Safdar $N$, Zahid $R$, Shah $S$ et al. Tuberculosis patients learning about second-hand smoke (TBLASS): results of a pilot randomised controlled trial. Int J Tuberc Lung Dis 2015; 19: 237-243

51 Altet-Gômez MN, Alcaide J, Godoy $P$ et al. Clinical and epidemiological aspects of smoking and tuberculosis: a study of 13,038 cases. Int J Tuberc Lung Dis 2005; 9: 430-436

52 Jee SH, Golub JE, Jo J et al. Smoking and risk of tuberculosis incidence, mortality, and recurrence in South Korean men and women. Am J Epidemiol 2009; 170: 1478-1485

53 Lin HH, Ezzati M, Chang HY. Association between tobacco smoking and active tuberculosis in Taiwan. Am J Respir Crit Care Med 2009; 180: $475-480$

54 Leung CC, Lam TH, Ho KS et al. Passive Smoking and Tuberculosis. Arch Intern Med 2010; 170: 287-292

55 Abal AT, Jayakrishnan B, Parwer $S$ et al. Effect of cigarette smoking on sputum smear conversion in adults with active pulmonary tuberculosis. Respir Med 2005; 99: 415-420

56 Maciel EL, Brioschi AP, Peres RL et al. Smoking and 2-month culture conversion during anti-tuberculosis treatment. Int J Tuberc Lung Dis 2013; 17: 225-228

57 Nijenbandring de Boer R, Oliveira e Souza Filho JB, Cobelens F et al. Delayed culture conversion due to cigarette smoking in active pulmonary tuberculosis patients. Tuberculosis (Edinb) 2014; 94: 87-91

58 d'Arc Lyra Batista J, de Fátima Pessoa Militão de Albuquerque M, de Alencar Ximenes $R A$ et al. Smoking increases the risk of relapse after successful tuberculosis treatment. Int J Epidemiol 2008; 37: 841 -851

59 Leung CC, Li T, Lam TH et al. Smoking and tuberculosis among the elderly in Hong Kong. Am J Respr Crit Care Med 2004; 170: 1027-1033

60 Thomas A, Gopi PG, Santha T et al. Predictors of relapse among pulmonary tuberculosis patients treated in a DOTS programme in South India. Inter J Tuberc Lung Dis 2005; 5: 5-25

61 Yen YF, Yen MY, Lin YS et al. Smoking increases risk of recurrence after successful anti-tuberculosis treatment: a population-based study. Int J Tuberc Lung Dis 2014; 18: $492-498$

62 Leung CC, Yew WW, Chan CK et al. Smoking adversely affects treatment response, outcome and relapse in tuberculosis. Eur Respir J 2015; 45: $738-745$

63 Gajalakshmi V, Peto R, Kanaka TS et al. Smoking and mortality from tuberculosis and other diseases in India: retrospective study of 43000 adult male deaths and 35000 controls. Lancet 2003; 362: 507-515

64 Peto R, Lopez AD. The future worldwide health effects of current smoking patterns. In: Koop CE, Pearson CE, Schwarz MR eds. Critical issues in global health. San Francisco, CA: Jossey-Bass; 2000

65 Jiménez-Ruiz CA, Andreas S, Lewis KE et al. Statement on smoking cessation in COPD and other pulmonary diseases and in smokers with comorbidities who find it difficult to quit. Eur Respir J 2015; 46: 61 -79

66 Bam TS, Aditama TY, Chiang CY et al. Smoking cessation and smokefree environments for tuberculosis patients in Indonesia - a cohort study. BMC Public Health 2015; 15: 604

67 Campbell IA, Chaudhary RD, Holdsworth GM et al. Brief advice to tuberculosis patients in Nepal to stop smoking: a pilot study by the Britain Nepal Medical Trust. Int J Tuberc Lung Dis 2014; 18: 1438-1442 
68 Louwagie GM, Okuyemi KS, Ayo-Yusuf OA. Efficacy of brief motivational interviewing on smoking cessation at tuberculosis clinics in Tshwane, South Africa: a randomized controlled trial. Addiction 2014; 109 : $1942-1952$

69 Zellweger JP, Cattamanchi A, Sotgiu G. Tobacco and tuberculosis: could we improve tuberculosis outcomes by helping patients to stop smoking? Eur Respir J 2015; 45: 583-585
70 El Sony A, Slama K, Salieh $M$ et al. Feasibility of brief tobacco cessation advice for tuberculosis patients: a study from Sudan. Int J Tuberc Lung Dis 2007; $11: 150-155$

71 Murray JF, Buist AS. Respiratory disorders related to smoking tobacco. In: Loddenkemper R, Kreuter M eds. The tobacco epidemic. ed 2, rev. and ext. Prog Respir Res. Basel: Karger; 2015; 42: 72-84

72 WHO End TB Strategy. Global strategy and targets for tuberculosis prevention, care and control after 2015.http://www.who.int/tb/ post2015_strategy/en/ 